\title{
Clinicopathological analysis of thymic malignancies with a consistent retrospective database in a single institution: from Tokyo Metropolitan Cancer Center
}

Yusuke Okuma ${ }^{1,5^{*}}$, Yukio Hosomi ${ }^{1}$, Kageaki Watanabe ${ }^{1}$, Yuko Yamada ${ }^{2}$, Hirotoshi Horio ${ }^{3}$, Yoshiharu Maeda ${ }^{4}$, Tatsuru Okamura ${ }^{1}$ and Tsunekazu Hishima ${ }^{2}$

\begin{abstract}
Background: Thymic epithelial tumors (TETs), which comprise thymoma and thymic carcinoma, are rare cancers with specific morphological and clinical features. Their clinical characteristics and outcomes have gradually been clarified by assessing large-scale, retrospective data obtained with international cooperation.

Methods: The study is a retrospective review of 187 Japanese patients with TETs who attended our institution from 1976 to 2012. Relevant clinical features of patients with TETs and their tumors, including histology, staging, treatment strategies, and overall survival, were investigated. Differences in survival were assessed by the Kaplan-Meier method and uni- and multi-variate Cox proportional hazards regression analyses.

Results: The 187 patients included 52 patients with stage I, 37 with stage II, 22 with stage III, and 76 with stage $\mathrm{IVa} / \mathrm{IVb}$ tumors according to the Masaoka-Koga Staging System. As to histological type, five patients had type A, 33 type AB, 19 type B1, 39 type B2, and 15 type B3 thymomas, whereas 68 patients had thymic carcinoma, including 11 with neuroendocrine carcinomas according to the 2004 WHO classification. Either insufficient data were available to classify the tumors of the remaining eight patients or they had rare types. Immunological abnormalities were present in 26 patients, most of whom had thymomas (21.8\% of the thymoma group). Most of the patients who presented with symptoms had myasthenia gravis or extensive thymic carcinoma. Secondary cancers were present in 25 patients (13.3\%). The overall 5- and 10-year survival rates for thymoma were 85.4 and $71.5 \%$, respectively, and those for thymic carcinoma were 33.8 and $2.3 \%$, respectively. OS differed significantly between stage IVa thymomas and thymic carcinomas. The stage and whether the tumors were thymomas or thymic carcinomas were significant determinants of survival according to multivariate analysis.
\end{abstract}

Conclusion: The efficacy of treatments for thymoma and thymic carcinoma should be investigated separately because these tumors differ in their clinical features and prognosis.

Keywords: Thymoma, Thymic carcinoma, Thymic epithelial tumor, World Health Organization classification, Treatment, Prognostic factor, Rare cancer

\footnotetext{
* Correspondence: y-okuma@cick.jp

${ }^{1}$ Department of Thoracic Oncology and Respiratory Medicine, Tokyo

Metropolitan Cancer and Infectious diseases Center Komagome Hospital,

3-18-22 Honkomagome, Bunkyo, Tokyo 113-8677, Japan

${ }^{5}$ Division of Oncology, Research Center for Medical Science, The Jikei

University School of Medicine, Minato, Tokyo, Japan

Full list of author information is available at the end of the article
} 


\section{Background}

Thymic epithelial tumors (TETs, or thymic malignancies) which comprise thymoma and thymic carcinoma, are rare cancers according to the definition of the RARECARE project, which is supported by the European Commission. Their annual incidence is approximately 0.15 cases in the United States [1] and 0.32 cases in the Netherlands [2] per 100,000 person-years. Thymic malignancies are extremely heterogeneous, with an exceedingly broad spectrum of morphological appearances and immunological abnormalities. Because thymomas are bioactive and have organotypic features that lead to autoimmune manifestations, whereas thymic carcinomas are not immunologically active and lack organotypic features, patients with thymic carcinoma usually have symptoms associated with tumor extension or metastasis.

Because of their rarity, the clinical characteristics and prognostic indicators in patients with thymic malignancies have not been well characterized [3]. Therefore, the International Thymic Malignancy Interest Group (ITMIG) was organized. Despite the paucity of evidence, this group has reached consensus agreements in support of some treatment modalities, having conducted some single-arm phase II studies and a few retrospective studies of small groups of treated patients with diverse backgrounds [4]. However, the optimal therapeutic strategy remains controversial. In previous studies, patients with thymoma and thymic carcinoma have basically received the same treatment. However, it has recently been suggested that the two types of tumors should be considered separate entities [5]. In addition, the ITMIG has proposed using the Masaoka-Koga staging system [6] and the 2004 World Health Organization (WHO) histological classification; both proposals have been accepted [7]. Thus, we believe it is necessary to review and clarify the nature and characteristics of these clinical entities in light of the proposed criteria. Furthermore, the National Comprehensive Cancer Network has updated its guidelines for the clinical management and treatment of thymic malignancies, despite their rarity [8].

The objective of the present study was to retrospectively clarify the clinical characteristics, prognosis, and prognostic indicators of patients with thymoma and thymic carcinoma according to the 2004 WHO classification [7] who had attended our institution over a 30-year period.

\section{Methods}

Database

This is a retrospective review of patients diagnosed with thymic malignancies between January 1976 and December 2012 identified from the databases at Tokyo Metropolitan Cancer and Infectious diseases Center Komagome Hospital (Tokyo, Japan). The codes of the International Classification of Diseases (9th edition) were used.
This retrospective study was approved by the Ethics Committee of the Tokyo Metropolitan Cancer and Infectious diseases Center Komagome Hospital (\#1049).

\section{Patients and histological evaluation}

A retrospective review of relevant clinical features and treatment-related data of 187 consecutive Japanese patients with diagnoses of thymic malignancies was performed. Their pathology was reviewed by a thoracic pathologist (TH) according to the 2004 WHO classification and Masaoka-Koga staging system [6]. Diagnoses of thymic carcinoma were confirmed by hematoxylin-eosin staining and immunohistochemistry for CD5 and/or CD117 (c-KIT) and/or p63 to exclude other malignant thoracic tumors, as well as supplemental testing for terminal deoxynucleotidyl transferase to distinguish carcinomas from thymomas. Clinical factors were also examined. Data were collected in accordance with the Standard Definitions and Policies of the ITMIG [4].

Clinical factors including age, sex, histological subtype, stage, immunological abnormalities, secondary malignancies, initial treatment -intent of modality, and survival were examined, relevant data having been obtained from medical records and laboratory data. Staging had been determined according to the Masaoka-Koga staging system by computed tomography, magnetic resonance imaging, positron emission tomography, or bone scanning. Histology was also classified according to the 2004 WHO classification. The patients had been treated with curativeintent or palliative-intent surgery, radiotherapy, chemotherapy, and best supportive care, or a combination of these modalities.

\section{Statistical analysis}

Descriptive statistics were used to summarize the patients' baseline characteristics. Survival time was defined as the period from the date of initiation of initial treatment (surgery, radiotherapy, chemotherapy, or best supportive care) to the date of death from any cause or last follow-up. The Kaplan-Meier method was used to assess overall survival and 5- and 10-year survival rates. Patients who had been lost to follow-up were censored at the time of last contact. These end points reflected clinical practice because of the retrospective nature of the data. In accordance with the ITMIG Standard Definitions and Policies, the 5-year survival rate of patients with thymic carcinomas and 10-year survival rate of those with thymoma were calculated. Correlations between histological subtype according to the 2004 WHO classification and Masaoka-Koga stage were evaluated using a nonparametric measure of statistical dependence between the two variables.

The log-rank test was used to identify prognostic indicators by uni- and multi-variate analysis. Candidate variables 
Table 1 Characteristics of patients and tumors in patients with thymic malignancies

\begin{tabular}{|c|c|c|c|c|c|}
\hline \multirow[t]{2}{*}{ Characteristics } & & \multicolumn{2}{|c|}{ Thymoma } & \multicolumn{2}{|c|}{ Thymic carcinoma } \\
\hline & & $n=119$ & $(\%)$ & $n=68$ & $(\%)$ \\
\hline Median age, & Years [range] & $58[26-81]$ & - & 63 [14-83] & - \\
\hline \multicolumn{6}{|l|}{ Gender } \\
\hline & Male & 52 & 43.7 & 38 & 55.9 \\
\hline & Female & 67 & 56.3 & 30 & 44.1 \\
\hline \multicolumn{6}{|l|}{ Histology } \\
\hline & Thymomas & 119 & 100.0 & & \\
\hline & Type A & 5 & 4.2 & & \\
\hline & Type B1 & 19 & 16.1 & & \\
\hline & Type B2 & 39 & 32.8 & & \\
\hline & Type B3 & 15 & 12.6 & & \\
\hline & Type AB & 33 & 27.7 & & \\
\hline & Other subtypes or missing data of thymoma & 8 & 6.7 & & \\
\hline & Thymic carcinomas & & & 68 & 100.0 \\
\hline & Squamous cell carcinoma & & & 46 & 67.6 \\
\hline & Mucoepidermoid carcinoma & & & 5 & 7.4 \\
\hline & Lymphoepithelioma-like carcinoma & & & 1 & 1.5 \\
\hline & Undifferentiated carcinoma & & & 3 & 4.4 \\
\hline & Neuroendocrine carcinomas & & & 11 & 16.2 \\
\hline & Small cell carcinoma & & & 3 & 4.4 \\
\hline & LCNEC & & & 2 & 2.9 \\
\hline & Carcinoid & & & 6 & 8.8 \\
\hline & Not classified in WHO classification & & & 2 & 2.9 \\
\hline
\end{tabular}

Staging

$\begin{array}{lcccc}\text { I } & 52 & 43.7 & 0 & 0 \\ \| & 31 & 26.1 & 6 & 8.8 \\ \text { III } & 12 & 10.1 & 10 & 14.7 \\ \mathrm{IVa} & 18 & 15.1 & 16 & 23.5 \\ \mathrm{IVb} & 6 & 5.0 & 36 & 52.9\end{array}$

Complications

$$
\begin{array}{lc}
\text { Immunological abnormalities (overlapped) } & 20 \\
\text { Myasthenia gravis } & 4 \\
\text { Pure red cell aplasia } & 5 \\
\text { Hypogammaglobulinemia } & 12
\end{array}
$$

Initial treatment-intent of modalities

Curative-intent treatment
Surgery
Surgery alone
Surgery with perioperative treatment
Radiotherapy
Definitive radiotherapy alone


Table 1 Characteristics of patients and tumors in patients with thymic malignancies (Continued)

\begin{tabular}{lllll}
\hline Chmoradiotherapy (sequential/concurrent) & 6 & 5.1 & 12 & 3.6 \\
Palliative-intent treatment & 3 & 2.5 & 25 \\
Chemotherapy alone & 2 & 1.7 & 24 \\
Best supportive care & 1 & 0.8 & 1 \\
\hline
\end{tabular}

analyzed included age (<70 vs. $\geq 70$ years), sex (male vs. female), staging, immunological abnormalities, secondary malignancies, and histological subtype according to the WHO classification 2004. Significance according to univariate analysis and multivariate Cox proportional hazard models was defined as $p<0.05$. All statistical analyses were performed using JMP9 (SAS Institute, Cary, NC, USA).

\section{Results}

Characteristics of patients with thymoma and thymic carcinoma and their tumors

Of the 187 patients, 119 (52 men, 67 women) had thymomas and 68 (38 men, 30 women) had thymic carcinomas. Their median age was 58 years for thymoma and 63 years for thymic carcinoma. As to histology, according to the 2004 WHO classification five patients had type A, 19 type B1, 39 type B2, 15 type B3, and 33 type $A B$ thymomas. Of the 68 patients with thymic carcinoma, 11 (16.2\%) had neuroendocrine carcinomas (three small cell carcinomas, two large cell neuroendocrine carcinomas, and six carcinoid tumors), 46 squamous cell carcinomas (67.6\%), five mucoepidermoid carcinomas (7.4\%), and one a lymphoepithelioma-like carcinoma. The remaining eight patients either had other histological types or relevant data were unavailable. Only a patient with thymic carcinoma had autoimmune-related manifestations. Most of the patients who presented with symptoms had myasthenia gravis or thymic carcinoma. Secondary malignancies were seen in 25 patients (13.4\%). At the time of diagnosis, 52 thymoma patients (43.7\%) had stage I, 31 (26.1\%) stage II, $12(10.1 \%)$ stage III, and $24(20.1 \%)$ stage IVa/ IVb according to the Masaoka-Koga Staging System, whereas six thymic carcinoma patients $(8.8 \%)$ had stage II disease, 10 (14.7\%) stage III disease, 16 (23.5\%) stage IVa disease, and 36 stage IVb disease (52.9\%). A variety of immunological paraneoplastic abnormalities were observed in the 26 patients with thymomas (21.8\%). There was some overlap among patients with immunological abnormalities.

Relevant patients' characteristics are summarized in Table 1. The median follow-up for all 187 patients at the time of analysis was 43.9 months (range: 0.3404.8 months).

\section{Treatment modalities and strategies for thymoma and} thymic carcinoma

Initial treatment was performed with curative-intent in $97.5 \%$ of patients with thymoma (surgery in $91.6 \%$, radiotherapy in $5.9 \%$ ) and in $63.2 \%$ of those with thymic carcinoma (surgery in $44.1 \%$, radiotherapy in $19.1 \%$ ).

The types of treatment modality are also summarized in Table 1.

\section{Clinical outcomes of thymoma and thymic carcinoma by stage and histological classification Stage}

The overall median OS of patients with thymoma was 235.2 months (95\% CI, 137.3-not reached), whereas that of those with thymic carcinoma was 32.4 months (95\% CI, $23.7-52.2)(p<0.0001)$ (Figure 1). The survival of patients with stages I, II, III, IVa, and IVb thymoma was not reached, not reached, 171.8, 110.1, and 83.8 months, respectively. The 5- and 10-year survival rates of patients with thymoma were 85.4 and $71.5 \%$, respectively (Figure $2 \mathrm{~b}-\mathrm{d}$ ). Conversely, survival of patients with stages II, III, IVa, and IVb thymic carcinoma was 78.9, 56.4, 27.3, and 21.7 months, respectively (Figure $2 \mathrm{~b}-\mathrm{d}$ ). The 5 - and 10year survival rates of patients with thymic carcinoma were 33.8 and $2.3 \%$, respectively.

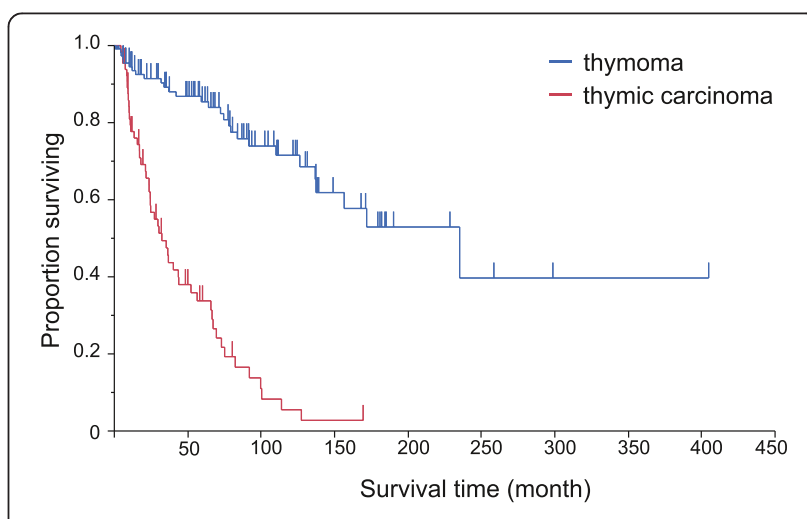

Figure 1 Kaplan-Meier curves showing median overall survival for thymoma ( $n=119)$ was 235.2 months $(95 \% \mathrm{Cl}, 137.3-$ not reached) and for thymic carcinoma $(n=68) 32.4$ months $(95 \%$ $\mathrm{Cl}, \mathbf{2 3 . 7 - 5 2 . 2 )}(\boldsymbol{p}<\mathbf{0 . 0 0 0 1})$. The 5 year-survival for thymoma and thymic carcinoma was 85.4 and $33.8 \%$, respectively. 


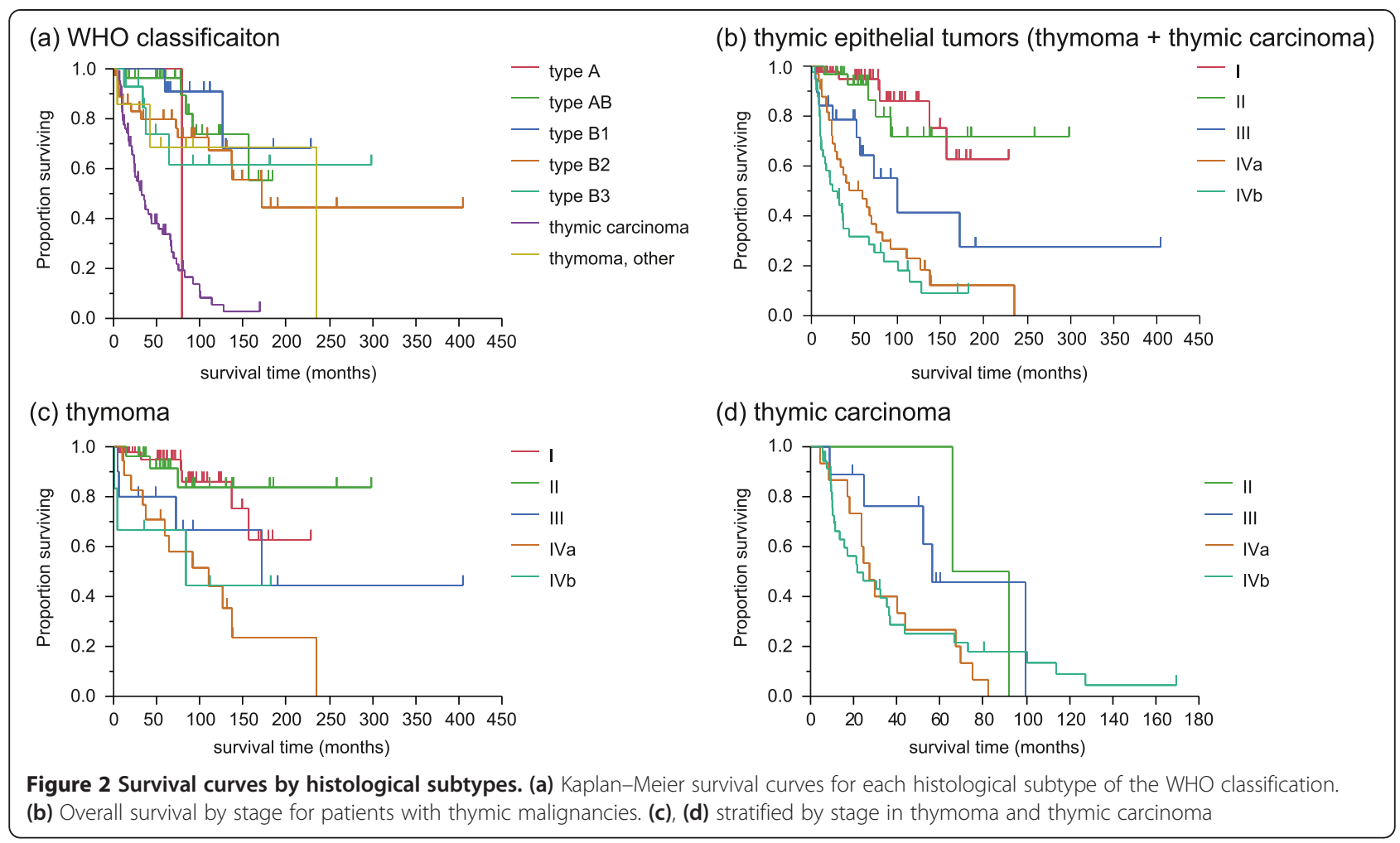

\section{Histological classification}

The 5- and 10-year survival rates were $100 \%$ and censored for patients with type A, 96.3 and $73.8 \%$ for those with type $A B, 90.9 \%$ and 68.2 for those with type B1, 79.8 and $67.3 \%$ for those with type B2, and 61.6 and $61.6 \%$ for those with type B3, respectively (Figure 2a). The median OS of patients with thymic carcinoma was 36.4 months (95\% CI, 23.7-52.2) for all stages combined, whereas the 5- and 10-year survival rates were 36.3 and $2.3 \%$, respectively. The median OS of patients with high-grade histology was 24.7 months, whereas that for patients with low-grade histology was 36.8 months. The overall median survival of patients with neuroendocrine carcinoma was 43.9 months. The median survival of the six patients with well-differentiated neuroendocrine carcinoma was 36.4 months (95\% CI 7.5-92.0) with a 5 -year survival of $20.0 \%$, whereas it was 43.9 months (95\% CI 5.6-127.4) in the five patients with poorlydifferentiated neuroendocrine carcinoma, with a 5 -year survival of $20.0 \%$.

\section{Correlation between tumor type according to the 2004 WHO classification and Masaoka-Koga stage}

The distribution of the WHO classification and MasaokaKoga stage of the 187 patients is shown in Table 2. The proportions of advanced stages (Masaoka-Koga stages III, IVa, and IVb) increased gradually from Type A thymoma to thymic carcinoma. There was a significant correlation between the WHO classification and Masaoka-Koga stage (Spearman's rank correlation coefficient $=0.69, p<0.0001$ ).

Prognostic factors affecting survival according to uni- and multi-variate analysis

According to univariate analysis, age and all MasaokaKoga stages were significantly correlated with survival in patients with thymoma. However, this was not the case in those with thymic carcinoma. According to multivariate analysis, early stages (Masaoka-Koga stage I or II) and advanced stages (IVa or IVb) of both thymomas and thymic carcinomas correlated significantly with survival (Table 3).

\section{Discussion and conclusion}

The present retrospective analysis examined the clinical outcomes of 187 patients with thymic malignancies. The clinical characteristics and outcomes in these unselected subjects were similar to those previously reported from large, multi-institutional series.

Based on the Müller-Hermelink classification [9], the WHO classification of thymomas was first proposed in 1999 [10]. In the 2004 WHO classification, thymic carcinoma, including neuroendocrine carcinoma, was separated from thymoma and given a new category [7]. Thymomas are classified into five groups: A, AB, B1, $B 2$, and B3. According to retrospective studies, Types A and $\mathrm{AB}$ have a better prognosis than $\mathrm{B} 1, \mathrm{~B} 2, \mathrm{~B} 3$, and 


\begin{tabular}{|c|c|c|c|c|c|c|c|c|c|}
\hline \multirow[t]{2}{*}{ WHO classification (2004) } & \multirow[t]{2}{*}{$\mathrm{N}^{\circ}$ of Pts } & \multirow[b]{2}{*}{ (\%) } & \multicolumn{5}{|c|}{ Masaoka-Koga Stages } & \multicolumn{2}{|c|}{ Survival rates (\%) } \\
\hline & & & $\mathrm{I}$ & II & III & $\mathrm{IVa}$ & $\mathrm{IVb}$ & 5-yr OS & 10-yr OS \\
\hline Thymoma & 119 & & 52 & 31 & 12 & 18 & 6 & 85.4 & 71.5 \\
\hline A & 5 & 2.7 & 4 & 1 & 0 & 0 & 0 & 100 & - \\
\hline$A B$ & 33 & 17.6 & 21 & 8 & 1 & 2 & 1 & 96.3 & 73.8 \\
\hline B1 & 19 & 10.2 & 11 & 5 & 0 & 2 & 1 & 90.9 & 68.2 \\
\hline B2 & 39 & 20.9 & 12 & 9 & 8 & 8 & 2 & 79.8 & 67.3 \\
\hline B3 & 15 & 8.0 & 1 & 5 & 3 & 5 & 1 & 61.6 & 61.6 \\
\hline Thymoma, other & 8 & 4.3 & 3 & 3 & 0 & 1 & 1 & 68.6 & 68.8 \\
\hline Thymic carcinoma & 68 & 36.4 & 0 & 6 & 10 & 16 & 36 & 33.8 & 2.3 \\
\hline Thymic carcinoma excluding NEC & 57 & 30.5 & 0 & 5 & 9 & 13 & 30 & 33.0 & 4.1 \\
\hline NETT & 11 & 5.9 & 0 & 1 & 1 & 3 & 6 & 27.3 & 9.1 \\
\hline$\overline{T o t a l}$ & 187 & 100.0 & 52 & 37 & 22 & 34 & 42 & 65.9 & 45.3 \\
\hline
\end{tabular}

WHO, World Health Organization; OS, overall survival; NETT, neuroendocrine thymic tumors.

carcinomas [11-13]. In contrast, other studies have failed to identify a correlation between survival and WHO classification [14-16]. These results were discussed as limitations owing to the difficulty in accurate reproducibility when diagnosing thymic malignancies with the WHO schema. However, clinical features such as immunological abnormalities and secondary malignancies may contribute to prognosis. Although our results did not demonstrate a significant association between abnormalities or secondary malignancies and survival, some studies have reported that types $\mathrm{A}$ and $\mathrm{AB}$ thymoma have a low association with myasthenia gravis, whereas types B1 and B2 are more likely to be associated with myasthenia gravis. Up to $45 \%$ of patients with thymoma develop myasthenia gravis $[17,18]$. According to the WHO classification, there are 13 subtypes of thymic carcinoma; $60-70 \%$ of all thymic carcinomas being subtypes of squamous cell carcinoma and lymphoepithelioma-like carcinoma. Recent biomarker investigations have explored c-KIT as a characteristic of thymic carcinoma [19]. Clinically, thymomas and thymic carcinomas have different patterns of recurrence: thymomas mainly result in pleural dissemination as opposed to the distant metastases characteristic of thymic carcinoma [20]. The WHO classification still has some limitations, in that distinguishing even thymoma and thymic carcinoma subtypes remains difficult. As to staging systems, the Masaoka-Koga staging system is widely accepted for both thymoma and thymic carcinoma, which is problematic because incorrect diagnoses, confounding of clinical entities, and intermingled management tend to occur. Till today, only a few studies according to the 2004 WHO Classification and Masaoka-Koga stage have been published (Table 4).
The present study is based on a relatively large database including all treated cases from the department of surgery, medical oncology, and radiation oncology. Additionally, we found that thymomas and thymic carcinomas exhibited a variety of clinical behaviors as reported in the past study. We believe that our single-institution data are reliable in that all cases were diagnosed by a pathologist who authored the thymic carcinoma section of the WHO classification book [7]. The lack of correlation between survival and WHO classification in thymomas may be attributable to the small numbers of patients studied, immunological abnormalities, or too few events because of the characteristically long survival. In our study, patients with thymomas had a similar prognosis to that previously reported, whereas both indolent and aggressive clinical courses occurred in patients with thymic carcinomas, including thymic squamous cell carcinoma. In neuroendocrine thymic tumors (or carcinomas) (NETT), the present small cohort of well-differentiated and poorly differentiated NETT showed similar clinical behavior to that reported in previous studies [21]. In the present study, no patients with NETT developed multiple endocrine neoplastic syndrome. As previously reported for NETT, the prognosis in this subgroup was poor [22]. The clinical entity of NETT is gradually becoming better known: the European Society of Medical Oncology has already published guidelines for NETT [23].

The key limitation of the present study was the small numbers of patients in each stage of thymoma or thymic carcinoma, resulting in a paucity of data compared with that obtained in randomized trials. However, this is a common limitation of studies of rare cancers. Second, we were unable to follow patients up, particularly young patients with thymoma or early stages of carcinoma whose 
Table 3 Uni- and multi-variate analysis of survival in patients with thymic malignancies

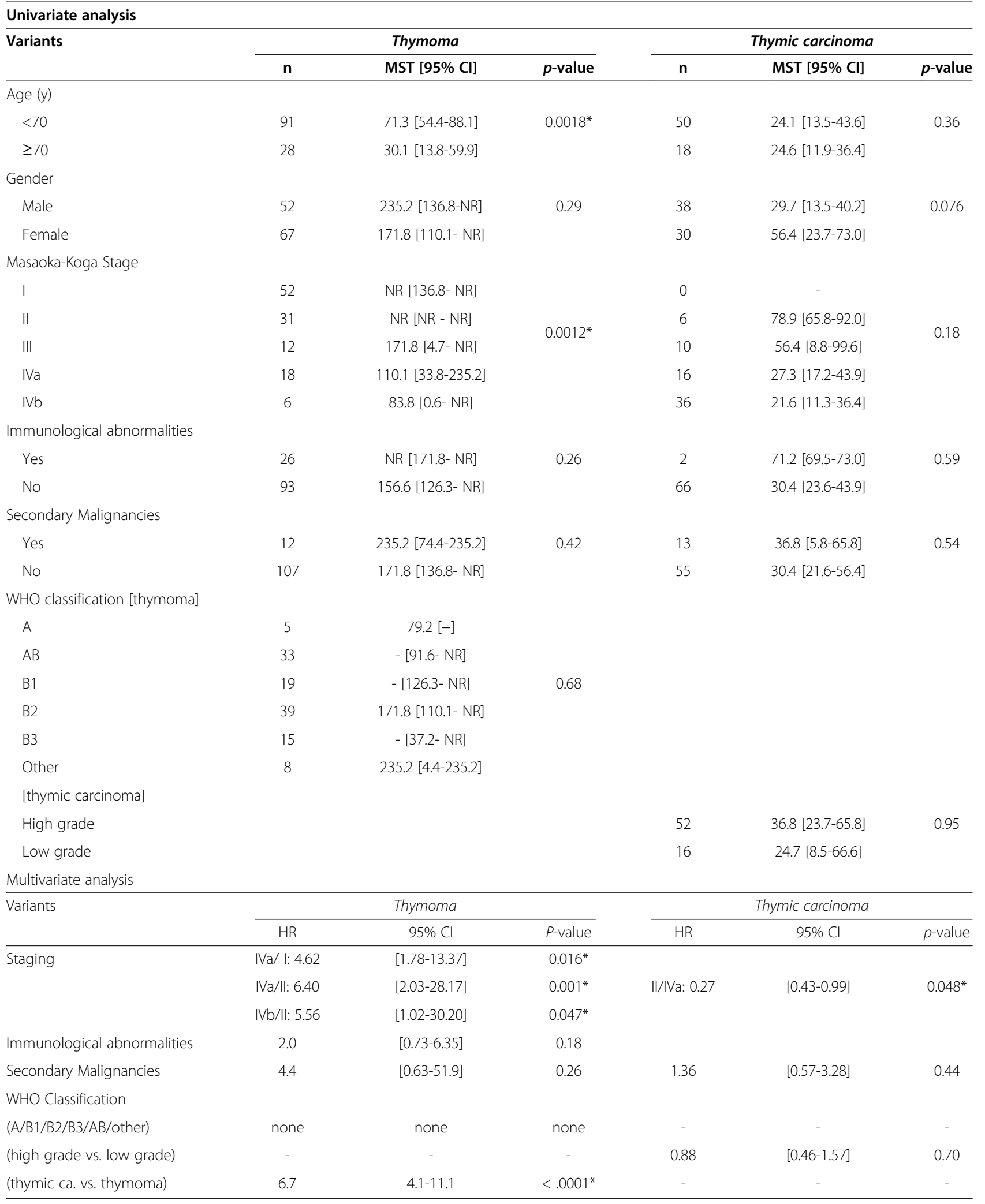

$\mathrm{NR}$, not reached; $\mathrm{Cl}$, confidence interval; $\mathrm{MST}$, median survival time; $\mathrm{HR}$, hazard ratio; $\mathrm{Cl}$ confidence interval; ${ }^{*} p<0.05$. 


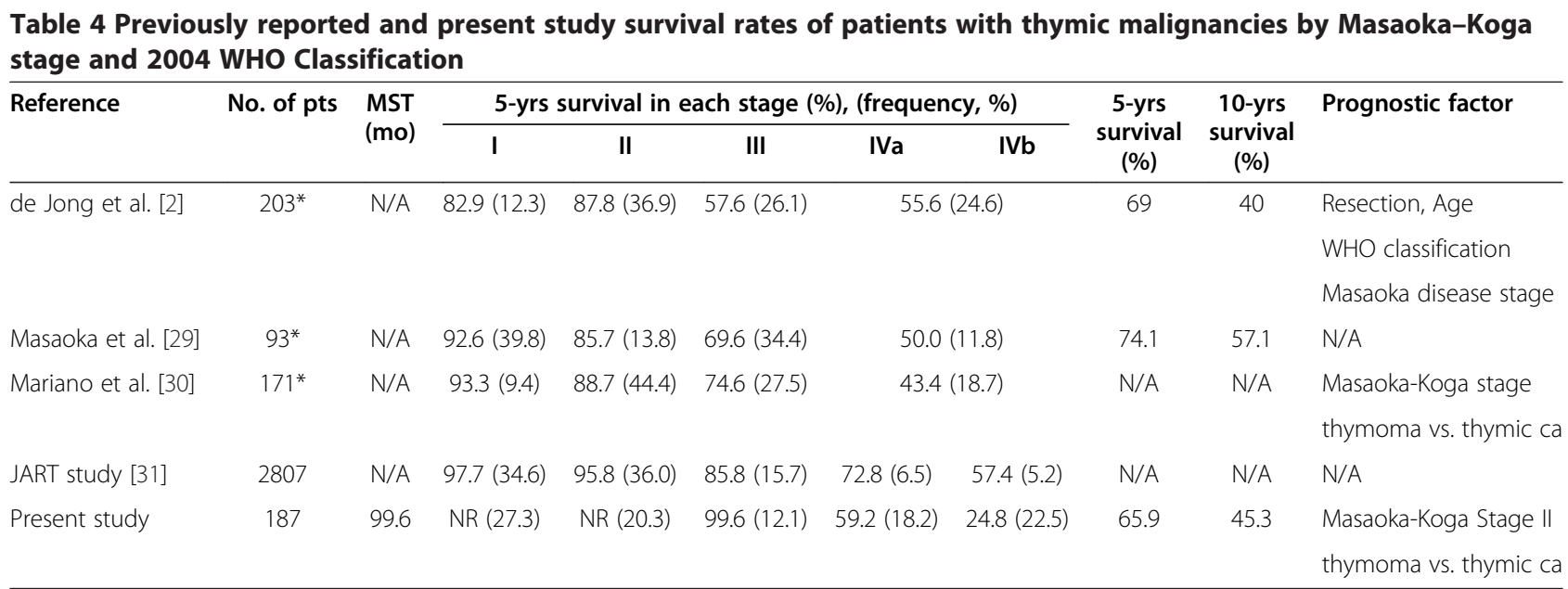

pts, patients; MST, median survival time; yrs, years; N/A, not available; *available data on survival is summarized.

tumors had been resected and who had no immunological abnormalities. Thus, there were more censored patients in the thymoma cohort than in the carcinoma cohort.

Large-scale databases are being established in Japan, the USA, and Europe as a first step toward conquering thymic malignancies. This approach appears to be a role model for studying rare diseases. Because these databases are drawn from surgical cases, they will provide little data on the clinical entities of thymic malignancies. Therefore, single-institution databases, such as that used in this study, are still meaningful because of the consistency of treatment and pathological evaluation; the latter would result in more reliable and reproducible diagnoses of thymic malignancies. Nevertheless unified, multi-institutional databases centered on the ITMIG are indispensable. Studies using such databases will clarify the clinical entities of and evolve treatment strategies for rare cancers such as thymic malignancies, which tend to fall behind in treatment development compared with common cancers. To minimize the biases from limited data concerning the reliability of diagnosis or treatment, every strategy must be carried out to overcome obstacles owing to the rarity of the cancer.

Plans are being made for prospective clinical trials on this rare cancer. However, the inevitably small sample size of future phase II studies will likely mean they have insufficient power to establish that findings are significant. In addition, as Weksler et al. have pointed out, a fundamental problem still remains in that the diagnosis of thymic malignancies, especially thymic carcinomas, is difficult [24]. In fact, in the WJTOG 4207L trial [25], $25 \%$ of patients diagnosed with thymic carcinoma by local hospitals were found to have incorrect diagnoses when centrally reviewed. Thus, central review of diagnoses is essential and the results of such studies must be interpreted with care. Investigators who plan clinical trials of thymic malignancies should incorporate central review by reliable pathologists who have experience with thymic malignancies. The importance of central review in clinical trials on rare cancers was demonstrated in the multi-institutional clinical trial of imatinib for c-Kit or platelet-derived growth factor receptor (PDGFR) positive sarcoma. In this trial, the concordance rate between the trial sites and central review for immunohistochemical staining was only 63.3\% [26]. Also, the guidelines for gastrointestinal stromal tumors (GISTs) recommend taking care with the diagnosis of c-Kit-negative GIST, which requires consulting a specialist in GISTs who has experience in additional antibody staining or $c$-Kit or PDGFR gene analysis $[27,28]$.

In summary, the further clinical management of thymoma and thymic carcinoma should be investigated separately because of the clinical differences between thymoma and thymic carcinoma. Moreover, a detailed populationbased series that highlights the many challenges clinicians face when treating thymic malignancies, for which little evidence-based data concerning therapy is available. Also, the advantages and disadvantages of a single-institutional database, especially on rare cancers, such as that used in this study, have been discussed. Although there have been advances in surgical techniques, radiation planning, systemic therapy, and supportive care for patients with thymic malignancies, more research and collaborative efforts are needed to produce evidence-based guidelines. International database projects and multidisciplinary meetings supported by the ITMIG will undoubtedly help fulfill this need.

\section{Ethics statement}

This study was approved by the Ethics Committee of Tokyo Metropolitan Cancer and Infectious diseases Center Komagome Hospital (Tokyo, Japan), and conducted in accordance with the Declaration of Helsinki. 


\section{Competing interests}

The authors declared that they have no competing interest.

\section{Authors' contributions}

$\mathrm{YO}, \mathrm{YH}$, and $\mathrm{KW}$ collected data and established a database for thymic malignancies. YO drafted the manuscript. $\mathrm{HH}, \mathrm{YM}$, and TO provided surgeons' and medical oncologists' perspectives. $Y Y$ and TH examined specimens of thymic malignancies and provided opinions from a pathology perspective. $\mathrm{YH}$ conceived of the study, participated in its design and coordination, and helped to draft the manuscript. All authors have read and approved the final manuscript.

\section{Acknowledgments}

The Authors thank Makoto Saito, the Senior Biostatistician in the Office for Clinical Research Support in Tokyo Metropolitan Cancer and Infectious diseases Center Komagome Hospital, for statistical advice.

This study was supported by a grant for Clinical Research from Tokyo Metropolitan Hospital.

\section{Note}

This study was presented at the European Cancer Congress 2013 Amsterdam, the Netherlands (P378).

\section{Author details}

'Department of Thoracic Oncology and Respiratory Medicine, Tokyo Metropolitan Cancer and Infectious diseases Center Komagome Hospital, 3-18-22 Honkomagome, Bunkyo, Tokyo 113-8677, Japan. ²Departments of Pathology, Tokyo Metropolitan Cancer and Infectious diseases Center Komagome Hospital, Bunkyo, Tokyo, Japan. ${ }^{3}$ Departments of Thoracic Surgery, Tokyo Metropolitan Cancer and Infectious diseases Center Komagome Hospital, Bunkyo, Tokyo, Japan. ${ }^{4}$ Department of Chemotherapy, Tokyo Metropolitan Cancer and Infectious diseases Center Komagome Hospital, Bunkyo, Tokyo, Japan. ${ }^{5}$ Division of Oncology, Research Center for Medical Science, The Jikei University School of Medicine, Minato, Tokyo, Japan.

Received: 1 December 2013 Accepted: 16 May 2014

Published: 20 May 2014

\section{References}

1. Engels EA, Pfeiffer RM: Malignant thymoma in the United States: demographic patterns in incidence and associations with subsequent malignancies. Int J Cancer 2003, 105(4):546-551.

2. de Jong WK, Blaauwgeers JL, Schaapveld M, Timens W, Klinkenberg TJ, Groen $\mathrm{HJ}$ : Thymic epithelial tumours: a population-based study of the incidence, diagnostic procedures and therapy. Eur J Cancer 2008, 44(1):123-130.

3. Engels EA: Epidemiology of thymoma and associated malignancies. J Thorac Oncol 2010, 5(10 Suppl 4):S260-S265.

4. Huang J, Detterbeck FC, Wang Z, Loehrer PJ Sr: Standard outcome measures for thymic malignancies. J Thorac Oncol 2010, 5(12):2017-2023.

5. National Comprehensive Cancer Network (NCCN): NCCN Clinical Practice Guidelines in Oncology. Thymomas and Thymic Carcinomas Version 1.2014. National Comprehensive Cancer Network 2013. Abstract available at http://www. nccn.org/professionals/physician_gls/pdf/thymic.pdf.

6. Masaoka A: Staging system of thymoma. J Thorac Oncol 2010, 5(10 Supple 4):S304-S312.

7. Travis W, Brambilla W, Müller-Hermelink H, Harris C: Chapter 3. Tumours of the Thymus. In World Health Organization classification of tumors. Pathology and genetics of tumors of the lung, pleura, thymus and heart. 3rd edition. Lyon: IARC press; 2004

8. Ettinger DS, Riely GJ, Akerley W, Borghaei H, Chang AC, Cheney RT, Chirieac LR, D’Amico TA, Demmy TL, Govindan R, Grannis FW Jr, Grant SC, Horn L, Jahan TM, Komaki R, Kong FM, Kris MG, Krug LM, Lackner RP, Lennes IT, Loo BW Jr, Martins R, Otterson GA, Patel JD, Pinder-Schenck MC, Pisters KM, Reckamp K, Rohren E, Shapiro TA, Swanson SJ: Thymomas and thymic carcinomas: clinical practice guidelines in oncology. J Natl Compr Canc Netw 2013, 11(5):562-576.

9. Muller-Hermelink HK, Marino M, Palestro G: Pathology of thymic epithelial tumors. Curr Top Pathol 1986, 75:207-268.

10. Rosai J, Sobin L: Histological Typing of Tumors of the Thymus. In WHO International Histological Classification of Tumours. 2nd edition. Berlin, Heidelberg: Springer-Verlag; 1999.
11. Okumura M, Ohta M, Tateyama H, Nakagawa K, Matsumura A, Maeda H, Tada H, Eimoto T, Matsuda H, Masaoka A: The World Health Organization histologic classification system reflects the oncologic behavior of thymoma: a clinical study of 273 patients. Cancer 2002, 94(3):624-632.

12. Kondo K, Yoshizawa K, Tsuyuguchi M, Kimura S, Sumitomo M, Morita J, Miyoshi T, Sakiyama S, Mukai K, Monden Y: WHO histologic classification is a prognostic indicator in thymoma. Ann Thorac Surg 2004, 77(4):1183-1188.

13. Rea F, Marulli G, Girardi R, Bortolotti L, Favaretto A, Galligioni A, Sartori F: Long-term survival and prognostic factors in thymic epithelial tumours. Eur J Cardiothorac Surg 2004, 26(2):412-418.

14. Rieker RJ, Hoegel J, Morresi-Hauf A, Hofmann WJ, Blaeker H, Penzel R, Otto HF: Histologic classification of thymic epithelial tumors: comparison of established classification schemes. Int J Cancer 2002, 98(6):900-906.

15. Suster S, Moran CA: Problem areas and inconsistencies in the WHO classification of thymoma. Semin Diagn Pathol 2005, 22(3):188-197.

16. Suster S, Moran CA: Thymoma classification: current status and future trends. Am J Clin Pathol 2006, 125(4):542-554.

17. Detterbeck FC, Parsons AM: Thymic tumors. Ann Thorac Surg 2004, 77(5):1860-1869.

18. Thomas CR, Wright CD, Loehrer PJ: Thymoma: state of the art. J Clin Oncol 1999, 17(7):2280-2289.

19. Petrini I, Zucali PA, Lee HS, Pineda MA, Meltzer PS, Walter-Rodriguez B, Roncalli M, Santoro A, Wang Y, Giaccone G: Expression and mutational status of c-kit in thymic epithelial tumors. J Thorac Oncol 2010, 5(9):1447-1453.

20. Huang J, Rizk NP, Travis WD, Riely GJ, Park BJ, Bains MS, Dycoco J, Flores RM, Downey RJ, Rusch WW: Comparison of patterns of relapse in thymic carcinoma and thymoma. J Thorac Cardiovasc Surg 2009, 138(1):26-31.

21. Gaur P, Leary C, Yao JC: Thymic neuroendocrine tumors: a SEER database analysis of 160 patients. Ann Surg 2010, 251(6):1117-1121.

22. Crona J, Bjorklund P, Welin S, Kozlovacki G, Oberg K, Granberg D: Treatment, prognostic markers and survival in thymic neuroendocrine tumours: a study from a single tertiary referral centre. Lung Cancer 2013, 79(3):289-293.

23. Oberg K, Hellman P, Ferolla P, Papotti M, Group EGW: Neuroendocrine bronchial and thymic tumors: ESMO Clinical Practice Guidelines for diagnosis, treatment and follow-up. Ann Oncol 2012, 23(Suppl 7):vii120-vii123.

24. Weksler B, Dhupar R, Parikh V, Nason KS, Pennathur A, Ferson PF: Thymic carcinoma: a multivariate analysis of factors predictive of survival in 290 patients. Ann Thorac Surg 2013, 95(1):299-303.

25. Takeda K, Hirai F, Yamanaka T, Taguchi T, Daga T, Shimizu J, Kogure Y, Kimura T, Tanaka K, Iwamoto Y, Ono A, Sasaki H, Fukuoka J, Nishiyama K, Seto T, Ichinose Y, Nakagawa K, Nakanishi Y, West Japan Oncology Group: A multicenter prospective study of carboplatin and paclitaxel for advanced thymic carcinoma: West Japan Oncology Group 4207L. J Clin Oncol 2013, 31(suppl; abstr 7529).

26. Sugiura H, Fujiwara Y, Ando M, Kawai A, Ogose A, Ozaki T, Yokoyama R, Hiruma T, Ishii T, Morioka H, Mugishima H: Multicenter phase II trial assessing effectiveness of imatinib mesylate on relapsed or refractory KIT-positive or PDGFR-positive sarcoma. J Orthop Sci 2010, 15(5):654-660.

27. Hirota S, Ohashi A, Nishida T, Isozaki K, Kinoshita K, Shinomura Y, Kitamura Y: Gain-of-function mutations of platelet-derived growth factor receptor alpha gene in gastrointestinal stromal tumors. Gastroenterology 2003, 125(3):660-667.

28. Heinrich MC, Corless CL, Duensing A, McGreevey L, Chen CJ, Joseph N, Singer S, Griffith DJ, Haley A, Town A, Demetri GD, Fletcher CD, Fletcher JA: PDGFRA activating mutations in gastrointestinal stromal tumors. Science 2003, 299(5607):708-710.

29. Masaoka A, Monden Y, Nakahara K, Tanioka T: Follow-up study of thymomas with special reference to their clinical stages. Cancer 1981, 48(11):2485-2492.

30. Mariano C, lonescu DN, Cheung WY, Ali RH, Laskin J, Evans K, Carolan H, Murray N: Thymoma: a population-based study of the management and outcomes for the province of British Columbia. J Thorac Oncol 2013, 8(1):109-117.

31. Kondo K: Report of ITMIG databese - Japan. J Thorac Oncol 2012, 7(11 suppl 5):S416.

\section{doi:10.1186/1471-2407-14-349}

Cite this article as: Okuma et al:: Clinicopathological analysis of thymic malignancies with a consistent retrospective database in a single institution: from Tokyo Metropolitan Cancer Center. BMC Cancer 2014 14:349. 